INVESTIGACIÓN EDUCATIVA

\title{
El programa de tutorias: canal de acompañamiento a las trayectorias educativas en medicina veterinaria
}

\author{
Fascendini PY*, Guaita ML, Ojeda N \\ Facultad de Ciencias Veterinarias, Universidad Nacional del Litoral (FCV-UNL), Argentina. \\ * Correspondencia: Paola Fascendini. FCV-UNL, RP Kreder 2805, 3080 Esperanza, Santa Fe, Argentina. \\ E-mail: pfascendini@fcv.unl.edu.ar
}

Recibido: 5 Diciembre 2019. Aceptado: 30 Junio 2020. Disponible en línea: 2 Diciembre 2020

Editor: R. Sobrero

\begin{abstract}
RESUMEN. El propósito del presente trabajo es socializar el impacto del Programa de Tutorías en la trayectoria educativa de los ingresantes a la carrera de medicina veterinaria de la Universidad Nacional del Litoral. El Programa de tutorías data del año 2010 cuando mediante el plan de mejoramiento de la carrera se instaura la necesidad de iniciar una labor de acompañamiento dirigida a los estudiantes ingresantes. Dicho programa se lleva a cabo mediante la labor profesional realizada desde la cátedra de Introducción a la Veterinaria, el Director de carrera y el Servicio de Orientación Educativa (S.O.E) de la Facultad de Ciencias Veterinarias siendo los coresponsables de dicha ejecución. El desafío constante del programa es responder a la construcción continua de alternativas que permitan a los estudiantes apropiarse de las habilidades necesarias para alcanzar el éxito académico. Teniendo en cuenta los resultados se observa que el Programa de Tutorías es recomendado por el $98 \%$ de los tutorados, debido a que el mismo es favorecedor de los procesos de afiliación universitaria. La novedad del programa es que se lleva a cabo por estudiantes avanzados, quienes luego de un proceso de selección y capacitación realizan la tarea orientativa.
\end{abstract}

SUMMARY. Tutorship programs: a way of accompanying educational trajectories in Veterinary Medicine. The purpose of this work is to socialize the impact of the Mentoring Program in the educational path of the entrants to the veterinary medicine career at the National University of the Coast. The Mentoring Program dates back to 2010 when, through the career improvement plan, the need to start an accompaniment work aimed at incoming students was established. This program is carried out through the professional work carried out by the Chair of Introduction to Veterinary Medicine, the Career Director and the Educational Guidance Service (S.O.E) of the Faculty of Veterinary Sciences, being the co-responsible for said execution. The constant challenge of the program is to respond to the continuous construction of alternatives that allow students to appropriate the necessary skills to achieve academic success. Taking into account the results, it can be seen that the Tutoring Program is recommended by $98 \%$ of the tutors, because it is favorable to the university affiliation processes. The novelty of the program is that it is carried out by advanced students, who after a selection and training process carry out the orientation task.

Palabras clave: tutorías de pares, acompañamiento, ingreso universitario, medicina veterinaria, trayectorias educativas, inclusión

Keywords: peer tutoring, accompaniment, university entrance, veterinary medicine, educational trajectories, inclusion

\section{Introducción}

Las universidades desarrollan nuevas estrategias académicas y pedagógicas tendientes a consolidar una estructura de oportunidades que permita a los estudiantes apropiarse de los conocimientos y habilidades necesarias para acceder al éxito académico, teniendo en cuenta que el ingreso a la educación universitaria en la Argentina está caracterizado por un público estudiantil cada vez más heterogéneo en términos de su perfil socioeconómico, educativo y en aspiraciones académicas y laborales (García, 2014). Entre estas actividades se instaura como pertinente el recorrido realizado por el Programa de tutorías desde su creación en el año 2010, por ser una instancia eficaz para el proceso de afiliación universitaria. La acción tutorial como papel central permite gestionar procesos de inclusión educativa no sólo en términos de acceso, permanencia y egreso sino también promueve las relaciones interpersonales. La educación tiene la misión de mediar entre el joven y el mundo (Arendt, 1993), de manera que se produzca la integración entre ambos, y permita a los estudiantes ser parte de él, sabiendo que el modo en que se lleva a cabo esta tarea será crucial para favorecer el proceso de adaptación al mundo de la cultura universitaria. La naturaleza social de los seres humanos no basta para adaptarlos a la vida organizada con otros seres, ya que se requieren de organizaciones sencillas y espacios que permitan la construcción de un hombre nuevo social en interacción con otros diferentes en un mundo heterogéneo y diverso (Durkheim, 1998). Por ello resulta primordial atender a estas necesidades mediante dispositivos institucionales que permitan acompañar el proceso del ingreso universitario, promoviendo así prácticas que hacen posible la 
inclusión socio-educativa y generan lazos que favorecen los procesos de adaptación.

En el marco de la Ley Nacional de Educación 26.206/06 y teniendo en cuenta el Plan de Mejora para la acreditación de la carrera de Medicina Veterinaria ante la CONEAU, en el mes de julio de 2010, se diseñó un programa de tutorías para ingresantes de la Facultad de Ciencias Veterinarias, en coordinación con el director de carrera, la cátedra de Introducción a la Veterinaria y el Servicio de Orientación Educativa, llevado a cabo por estudiantes de años superiores.

El proyecto de las tutorías surge como una respuesta a las necesidades de los estudiantes que requieren ser andamiadas en el proceso de formación. El Programa está orientado a acompañar el ingreso estudiantil y guiar a los nuevos estudiantes en el proceso de adaptación universitaria, con el fin de que se adquieran y desarrollen las habilidades y competencias requeridas para alcanzar una eficaz adaptación al ámbito de la educación superior.

Las tutorías son desarrolladas por estudiantes avanzados de la carrera a los cuales se les designa un grupo de 15 estudiantes ingresantes con la coordinación de la cátedra de Introducción a la Veterinaria y S.O.E. (Servicio de Orientación Educativa) de la FCV. El Programa se asienta en una metodología experiencial que articula acciones a través de un coordinador de tutores (docente de la cátedra de Introducción a la Veterinaria), el que acompaña la intervención, apoyado por el S.O.E. que cumple la misión de colaborar y personalizar la escucha de las problemáticas individuales, a la vez que vierte orientaciones específicas a las necesidades que aquejan a cada estudiante en particular (Fascendini, 2016). El S.O.E realiza tareas de acompañamiento estudiantil desde el ingreso universitario hasta el egreso, ofreciendo espacios de orientación educativa que permitan brindar las herramientas necesarias para favorecer el aprendizaje universitario.

El para qué del programa prioriza la necesidad de incorporación de la tutoría como una estrategia permanente que colabora en los procesos iniciales del ingreso universitario permitiendo una más ajustada afiliación al mundo superior. Para poder evaluar la funcionalidad del programa se ha diseñado una encuesta valorativa junto a entrevistas individuales, que permiten recoger no sólo datos cuantitativos sino también cualitativos en el ejercicio del rol, tanto de los tutores como de los tutorados, considerando especialmente el desarrollo correspondiente a la cohorte 2018 en esta comunicación.

Los objetivos generales del Programa de tutorías son: I) Favorecer la incorporación de los ingresantes de Medicina Veterinaria a la vida universitaria como una estrategia permanente que ayude a disminuir el desgranamiento de estudiantes; II) Revalorizar el vínculo personal y el aprendizaje en grupo como instancia válida para mejorar el rendimiento académico en el nivel universitario. III) Establecer a la tutoría como referente relacional facilitadora de la integración académica social de los ingresantes.

Los objetivos específicos del Programa de Tutorías son: I) Mejorar el desempeño de los estudiantes a través de acciones que apunten a construir herramientas metacognitivas; II) Acompañar a los ingresantes detectando aquellos que se encuentran en una situación límite o crítica.

\section{Materiales y métodos}

Una de las novedades del Programa es que está integrado dentro de la planificación de la asignatura Introducción a la Veterinaria (primer semestre de la carrera) donde los alumnos ingresantes deben cumplimentar un mínimo de $70 \%$ de asistencia a los encuentros programados para poder acceder a la promoción de la asignatura. De esta manera se logra posicionar al programa en un lugar estratégico para acompañar el proceso de ingreso universitario. Además, los tutores deben realizar un registro semanal de las actividades realizadas en los encuentros tutoriales, las que formarán parte del proceso evaluativo para obtener la calificación final de la asignatura. Esto permite conocer en profundidad las dificultades observadas en los estudiantes y se convierte en una instancia preventiva. Los tutores tienen a su cargo grupos de trabajo conformados por los tutorados, quienes son asignados por el coordinador, habiendo realizado con antelación entrevistas de selección y conocimiento de las aptitudes de los tutores, con el fin de ajustar el personal a los objetivos de trabajo. Las aptitudes evaluadas para el ejercicio del rol se circunscriben al liderazgo, creatividad, habilidad verbal, tipo de motivación, relaciones interpersonales y comunicatividad. En el marco del Programa de Tutorías y en la instancia de selección de tutores se previó la aplicación de un dispositivo de evaluación de los aspirantes. Este permite dar cuenta de los elementos que sirven a la hora de la selección, siendo su objetivo principal evaluar a los estudiantes más competentes para el ejercicio del rol tutorial. La convocatoria estuvo dirigida a estudiantes avanzados en la carrera, debiendo reunir ciertos requisitos formales tales como tener el $75 \%$ de las asignaturas del primer año aprobadas y regularizadas, el $80 \%$ del Ciclo Básico de la carrera de Medicina Veterinaria, como mínimo y contar con un desempeño académico satisfactorio en el último año, además de otros requerimientos vinculados a la presentación de antecedentes y curriculum, sumado a una valoración cualitativa de aptitudes para el ejercicio del rol tutorial.

La selección se realizó en dos etapas, la primera consistió en el análisis del curriculum de los aspirantes para luego de su lectura, llevar a cabo entrevistas focalizadas y semiestructuradas en la que se aplicó una serie de técnicas en habilidades sociales para obtener el perfil individual más acorde con la función solicitada. La segunda etapa consistió en la tabulación de los datos 
obtenidos y la elaboración de una nómina jerárquica de tutores para el ejercicio de dicho rol, con el propósito de que nadie quede fuera de las instancias de capacitación, ya que en un futuro los que han quedado fuera del orden de mérito vuelven a inscribirse en la siguiente convocatoria y así se van instrumentando acciones de fortalecimiento y formación de los recursos humanos.

Los tutores realizan encuentros semanales donde desarrollan actividades previamente programadas en reuniones de coordinación y mediatizadas por material planificado teniendo en cuenta las necesidades y demandas vertidas. Estas necesidades son el resultado de la valoración que cada tutor realiza de su grupo de tutorados, detectando en el intercambio y encuentro grupal aquellos emergentes que requieren ser abordados. En paralelo con los encuentros tutoriales, los tutores asignados al programa se reúnen quincenalmente con el coordinador, donde se organizan las actividades, se plantean las sugerencias e inconvenientes suscitados a la hora del trabajo grupal con los tutorados, se analizan las problemáticas emergentes, buscando una constante adaptación a las necesidades estudiantiles y a cada grupo en particular.

Desde el S.O.E, en trabajo conjunto con el coordinador de las tutorías y docentes que conforman la cátedra de Introducción a la Veterinaria, se ofrecen espacios alternativos para los tutores quienes acercan en forma quincenal las necesidades particulares, que son vertidas como un modo de reflexionar conjuntamente los procesos que van emergiendo en todo trabajo grupal, sabiendo que el mismo es una construcción que requiere de intervenciones frecuentes que hacen al seguimiento del tutor.

Los contenidos se planifican para cada encuentro teniendo en cuenta los pedidos estudiantiles. Los temas más ranqueados se vinculan con el saber organizarse para estudiar, el conocer los trámites institucionales de inscripción y manejo administrativo, las estrategias de estudio, el interés y motivación, la contención afectiva y la producción de textos académicos, entre otros. Los temas abordados se relacionan con estrategias metacognitivas, organización, planificación para el cursado, lectura e interpretación de textos académicos, análisis del reglamento de enseñanza y plan de estudio, manejo del Sistema de Información Universitaria - Guaraní, rol del médico veterinario, riesgos profesionales y medidas de bioseguridad durante el cursado de la carrera. EI S.O.E al ser un espacio de acompañamiento estudiantil orienta acciones que permiten favorecer la afiliación universitaria y atender a las necesidades que los mismos tutores comparten, tanto desde ellos mismos, como de sus tutorados, por lo que se convierte en un espacio que como verbalizan los estudiantes es "el lugar donde podemos aprender a ser nosotros mismos".

Se llevan a cabo tres talleres formativos, en el segundo cuatrimestre del año académico, a cargo del S.O.E, do- centes de la cátedra de Introducción a la Veterinaria y Director de carrera, los que tienen como propósito ofrecer herramientas personales a quienes se encuentran formándose para dicho rol. La capacitación ofrecida apunta al fortalecimiento de habilidades requeridas para el ejercicio del rol tutorial y se circunscribe a los siguientes aspectos: capacidad de liderazgo (capacidad para tener personas a cargo, motivarlos y dirigirlos hacia un objetivo, capacidad de mando y estilo para lograrlo); creatividad y resiliencia, capacidad para brindar respuestas novedosas, originales, flexibilidad en las respuestas y en la resolución de problemas; habilidad verbal (capacidad para comunicarse de manera oral o escrita con los interlocutores, capacidad para hacerse comprender, adecuación del vocabulario al medio en el cual se desenvuelve); modalidad de pensamiento, analítico, sintético, capacidad para encontrar soluciones adecuadas al medio y resolver problemas que implican el uso del razonamiento; motivación para el puesto (grado de interés demostrado en el puesto de trabajo, deseo puesto en el trabajo específico y en general en el desarrollo laboral); relaciones interpersonales (modalidad de contacto con las demás personas, capacidad de empatía, introversión o extraversión, identificación con los demás, comunicatividad). En los talleres de formación se propició un clima de encuentro, confianza, aceptación y aprendizaje, buscando un cambio de paradigma pedagógico donde el eje central se encuentra en el protagonismo del tutor y sus necesidades más troncales.

Se diseñó un cuadernillo de trabajo articulado con diversas temáticas las que estàn divididas en ejes relacionados con procesos adaptativos, organización personal y de estudio, canales de aprendizaje, estrategias metacognitivas, inserción en la universidad, sistemas evaluativos, tipos de exámenes entre otros. La característica central del material es su funcionalidad y autoaplicabilidad ya que consta no sólo de una atractiva presentación visual por el uso de colores e imágenes, sino que además ofrece, recursos multimedia insertos en el mismo texto a modo de link, enriquecidos por ejercicios de auto aplicación que permiten favorecer el autoconocimiento en las temáticas mencionadas. Los encuentros semanales que llevan a cabo los tutores con sus tutorados, suelen ser de 90 minutos, donde la actividad se centraliza en generar un espacio participativo que favorezca el debate, la escucha, la confianza, y la posibilidad de verter y canalizar las preocupaciones que emerjan ante este nuevo espacio formativo. Además presenta actividades de autorreflexión y autoanálisis de las propias maneras y formas de apropiación de la información con pautas y orientaciones específicas para cada caso en particular, resultando un formato práctico y útil a las necesidades estudiantiles.

La instancia evaluativa del Programa de Tutorías llevada a cabo persiguió como objetivo primordial el reconocimiento de logros y obstáculos en la puesta en marcha de éste espacio alternativo a los de enseñanza formal, como así también conocer la vivencia que han 
tenido tanto tutores como tutorados en el ejercicio del rol. Para ello se diseñó una encuesta valorativa que permitió recoger datos en función de la evaluación del ejercicio tutorial, y analizar también las voces y vivencias de las experiencias estudiantiles mediante entrevistas semiestructuradas, para dar cuenta del significado otorgado a este dispositivo institucional.

El diseño de la encuesta se organizó en el formato de documento drive para facilitar su aplicación y fue administrado a 126 estudiantes ingresantes a la carrera de Medicina Veterinaria, quienes han participado de los encuentros tutoriales en el primer semestre de 2018. La encuesta consta de una serie de interrogantes dirigidos a la funcionalidad del programa de tutorías, a la necesidad de brindar continuidad al mismo, a la aplicabilidad de las temáticas enseñadas y a la experiencia realizada.

Los tutores acercan a los tutorados un link que los conduce a una encuesta valorativa para poder verter la opinión de los mismos, en torno a la tarea compartida. Además los tutores realizan entrevistas personales con el S.O.E con el objetivo de conocer cuáles han sido los logros u obstáculos en el ejercicio del rol tutorial.

\section{Resultados}

Teniendo en cuenta las respuestas brindadas, se hace referencia que en un $92,9 \%(117 / 126)$ los tutorados enfatizan la necesidad de darle continuidad a este programa, siendo que los aportes que han recibido de la experiencia ha resultado ser un espacio que les permitió aclarar dudas, favorecer su organización para la vida universitaria, ser un apoyo y acompañamiento favorable para los procesos del ingreso, potenció las estrategias de estudio, resultando en síntesis una guía y referente positivo.

En relación con el ítem referente a las actitudes evidenciadas en el tutor expresan que el 79,4\% (100/126) de los tutores se ha manifestado dispuesto, el $73,8 \%$ (93/126) responsable, el $70,6 \%(89 / 126)$ abierto a responder a las necesidades, el 68,3\% (86/126) comprometido, resaltando valores primordiales en el trato humano y cercano que propician en el intercambio con el otro. Además resaltan la amabilidad, clarificación de las dudas, confiabilidad, simpatía, puesta al servicio de su función y enfatizan la colaboración constante que el S.O.E ha brindado en toda esta labor.

En relación con las temáticas desarrolladas en los encuentros tutoriales y el grado de conformidad de los tutorados expresan que el $44,4 \%(56 / 126)$ ha estado muy conforme y el $49,2 \%$ (62/126) conforme. Teniendo en cuenta el ítem temáticas de los encuentros, han referido aplicabilidad de las estrategias de estudio y organizativas enseñadas en el $54 \%(68 / 126)$ frecuentemente, evidenciando utilidad de lo comunicado.
Las valoraciones cualitativas a partir de la pregunta cómo definirían a la tutoría luego de haber experimentado el programa, los ingresantes la conceptualizan como una experiencia maravillosa en la que se puede hacer amigos, conocer compañeros, expresarse, perdiendo el miedo a lo nuevo, constituyendo una guía primordial para el ingreso, sabiendo del valor que tiene contar con alguien incondicional, siempre presente para cualquier duda o temor, una forma de aprender a trabajar en grupo, interesante, un consejero, una persona que siempre te alienta a seguir, entre otras menciones que verbalizan y refieren en torno a la labor del tutor.

Los resultados de la encuesta valorativa del Programa de Tutorías destacan las siguientes consideraciones: I) Las Tutorías proporcionan información y aclaran tus dudas sobre el plan de estudios: $89 \%$ contestó mucho, $10 \%$ regular, $1 \%$ poco, $0 \%$ nada; II) Las Tutorías amplían tu visión sobre el ámbito profesional y laboral: 64\% contestó mucho, $29 \%$ regular, $6 \%$ poco y $1 \%$ nada; III) Las Tutorías proporcionan información y aclarar tus dudas sobre aspectos académicos administrativos: $70 \%$ contestó mucho, $26 \%$ regular, $4 \%$ poco y $0 \%$ nada; IV) Las Tutorías promueven la participación y asistencia a eventos culturales, recreativos, sociales y académicos: $45 \%$ contestó mucho, $41 \%$ regular, $11 \%$ poco y $4 \%$ nada; V) Las Tutorías, favorecen la adaptación al mundo universitario: $78 \%$ contestó mucho, $21 \%$ regular, $1 \%$ poco y $0 \%$ nada; VI) Las Tutorías favorecen la adquisición de habilidades que permiten mejorar el rendimiento académico: $67 \%$ contestó mucho, $29 \%$ regular, $4 \%$ poco y $1 \%$ nada.

Además los alumnos que han realizado esta experiencia recomiendan la continuidad del programa en un $98 \%$ ya que lo reconocen como un sistema que permite colaborar en sus procesos adaptativos.

Otra lente con la cual se ha mirado a la tutoría, han sido las palabras de los mismos tutores. En la puesta en marcha de este dispositivo, se promovieron espacios personales de análisis y valoración de la propia experiencia, ofrecidos desde el S.O.E, como un modo introspectivo de facilitar el enriquecimiento personal. A través de entrevistas individualizadas se promovió el diálogo para analizar las emociones y la experiencia construida. Los tutores refieren que ha habido un antes y un después de las tutorías en su experiencia estudiantil, pues esta incursión les ha posibilitado un incremento de las propias habilidades comunicacionales, ha mejorado la confianza en sí mismos, potenciando su autoestima y favoreciendo además el aprendizaje con otros; sabiendo que en el ejercicio de esta función han consolidado su crecimiento personal, construyendo su rol como estudiantes al servicio de otros, como actores sociales activos en el ámbito universitario. En su gran mayoría desean repetir la experiencia, como un modo de ir ensayando roles ocupacionales, pero fundamentalmente como una manera de forjar mejores habilidades en la conformación de sujetos íntegros. 


\section{Conclusiones}

El ingreso a la universidad se ve atravesado por variables diversas, que van condicionando el proceso de afiliación de los estudiantes. Nuestros esfuerzos por acompañar a los estudiantes en este ingreso, encuentran en el Programa de Tutorías el dispositivo adecuado para atenuar el impacto de la transición entre el nivel medio y el nivel superior, por estar enmarcado en una materia introductoria, y ser un requisito de aprobación, se convierte en una herramienta que integrada al programa de estudios permite un acompañamiento personalizado.

La puesta en marcha de esta estrategia institucional tiende a favorecer en los ingresantes la adquisición de herramientas fundamentales para el proceso de aprendizaje mejorando las habilidades personales y la puesta en práctica de las mismas.

Se rescata como necesario e indispensable el rol tutorial, por los intercambios interpersonales que ponen a prueba aptitudes, habilidades, rasgos en la personalidad que permiten madurar psico-afectivamente, pulir asperezas caracterológicas y propiciar espacios de reflexión internos valiosos para la formación humana

Los alcances del Programa de Tutorías como un dispositivo preventivo, ha sido por momentos controversial, debido a los insumos requeridos, lo real, es que es un dispositivo concreto y posible que continúa su accionar en forma sistemática, haciendo concreta la permanencia de muchos de los estudiantes que se incorporan al mundo universitario. Lo refrendan los protagonistas: los ingresantes lo recomiendan porque ha favorecido su proceso de afiliación; los tutores lo hacen porque buscan transmitir su experiencia y devolver a través de este servicio, lo que ellos mismos recibieron de la Universidad.

Así visto, el Programa de Tutorías se transforma en una experiencia pedagógica original, que se lleva a cabo entre pares que tienen un mismo objetivo. superarse.

Apostar a estos jóvenes significa acompañarlos con propuestas concretas, fortaleciendo la esperanza de un país creciente, que requiere voluntades acérrimas y sedientas de oportunidades. Ratificamos la instrumentación del Programa de Tutorías como una propuesta que permite resignificar el lugar del sujeto y otorga espacios de mediación para favorecer, no solo el ingreso, sino también la permanencia en la elección vocacional de los estudiantes universitarios.

\section{Agradecimientos}

A todos los tutores participantes. A los tutorados. Al equipo de cátedra.

\section{Bibliografía}

Arendt H. 1993. La condición humana. Editorial Paidós, Barcelona. $36 \mathrm{pp}$.

Badillo Guzmán J. 2007. La tutoría como estrategia viable de mejoramiento de la calidad de la educación superior. Reflexiones en torno al curso CPU-e. Revista de Investigación Educativa 5: 1-22

Durkheim E. 1998. Educación y pedagogía. Ensayos y controversias. Editorial Losada, Bs. Aires, Argentina. 218 pp.

García AM. 2014. Rendimiento académico y abandono universitario, modelos, resultados y alcances de la producción académica en la Argentina. Revista Argentina de Educación Superior 8: 9-38.

Fascendini PY. 2016. Estrategias metacognitivas: el ingreso a la universidad. ¿Cómo aprende el que aprende? Editorial Académica Española. 112 pp.

Gismero González E. 2000. Escala de habilidades sociales. Tea Ediciones, Madrid. 4 pp. 